\title{
The Shore Point Existence Problem is Equivalent to the Non-Block Point Existence Problem
}

\author{
Daron Anderson Trinity College Dublin. Ireland \\ andersd3@tcd.ie Preprint February 2019
}

\begin{abstract}
We prove the three propositions are equivalent: (a) Every Hausdorff continuum has two or more shore points. (b) Every Hausdorff continuum has two or more non-block points. (c) Every Hausdorff continuum is coastal at each point. Thus it is consistent that all three properties fail. We also give the following characterisation of shore points: The point $p$ of the continuum $X$ is a shore point if and only if there is a net of subcontinua in $\{K \in C(X): K \subset \kappa(p)-p\}$ tending to $X$ in the Vietoris topology. This contrasts with the standard characterisation which only demands the net elements be contained in $X-p$. In addition we prove every point of an indecomposable continuum is a shore point.
\end{abstract}

\section{Introduction}

Leonel [11] has improved the classic non-cut point theorem of Moore [13] by showing every metric continuum has two or more shore points. Bobok, Pyrih and Vejnar [6] observed Leonel's two shore points have the stronger property of being non-block points.

In [2] the author proved it is consistent the result fails to generalise to Hausdorff continua. Under Near Coherence of Filters (NCF) the Stone-Čech remainder $\mathbb{H}^{*}$ of the half-line lacks nonblock points and hence lacks coastal points.

This left open the question of whether there is a consistent example of a Hausdorff continuum without shore points. This paper gives a positive answer. Indeed we show the shore point and non-block point existence problems are equivalent. They are also equivalent to a number of other problems involving shore, non-block, and coastal points of Hausdorff continua.

We also prove every shore point $p \in X$ has the stronger property of being a proper shore point. That means there is a net of subcontinua in the hyperspace $\{K \in C(X): K \subset \kappa(p)-p\}$ tending to $X$ in the Vietoris topology. This is not apparent from the definition of a shore point, which only requires the net elements be contained in $X-p$. 


\section{Terminology and Notation}

For sets $A$ and $B$ define $A-B=\{a \in A: a \notin B\}$. For $B=\{b\}$ we write $A-b$ without confusion. For $A \subset B$ we do not presume $A$ is a proper subset of $B$. For a subset $S \subset X$ denote by $S^{\circ}$ and $\bar{S}$ the interior and closure of $S$ respectively. The boundary of $S$ means the set $\partial S=\bar{S} \cap \overline{(X-S)}$.

Throughout $X$ is a continuum. That is to say a nondegenerate compact connected Hausdorff space. For background on metric continua see [10] and [14]. The results cited here have analagous proofs for non-metric continua.

Throughout all maps are assumed to be continuous. The map $f: X \rightarrow Y$ of continua is called monotone to mean $f^{-1}(y) \subset X$ is connected for each $y \in Y$. Theorem 6.1.28 of [8] says moreover $f^{-1}(K) \subset X$ is a continuum for each subcontinuum $K \subset Y$.

For $a, b \in X$ we call $X$ irreducible about $\{a, b\}$ to mean $\{a, b\}$ is not contained in a proper subcontinuum of $X$. The subspace $A \subset X$ is called a semicontinuum to mean for each $a, c \in A$ some subcontinuum $K \subset A$ has $\{a, c\} \subset K$. Every subspace $A \subset X$ is partitioned into maximal semicontinua called the continuum components of $A$.

For $N \geq 2$ we say the subcontinua $X_{1}, \ldots, X_{N} \subset X$ form a decomposition and write $X_{1} \oplus$ $\ldots \oplus X_{N}$ to mean $X_{1} \cup \ldots \cup X_{N}=X$ and no $X_{n}$ is contained in the union of the others. We call $X$ decomposable to mean it admits a decomposition and indecomposable otherwise. The latter is equivalent to admitting no decomposition with $N=2$ and equivalent to each proper subcontinuum being nowhere dense. We say $X$ is hereditarily indecomposable to mean its every subcontinuum is indecomposable. Equivalently each pair of subcontinua are either disjoint or nested.

The composant $\kappa(x)$ of the point $x \in X$ is the union of all proper subcontinua that have $x$ as an element. Indecomposable metric continua are partitioned into $\mathfrak{c}$ many pairwise disjoint composants [12]. In case $\kappa(x) \neq \kappa(y)$ then $X$ is irreducible about $\{x, y\}$.

By boundary bumping we mean the principle that, for each proper closed $E \subset X$, each component $C$ of $E$ meets the boundary $\partial E=\bar{E} \cap \overline{X-E}$. For the non-metric proof see $\S 47$, III Theorem 2 of [10]. One corollary of boundary bumping is that any $p \in X$ is in the closure of each continuum component of $X-p$.

Throughout $C(X)$ is the set of subcontinua of $X$. We call $p \in X$ a shore point to mean for each finite collection of open sets $U_{1}, U_{2}, \ldots U_{n} \subset X$ some subcontinuum $K \subset X-p$ meets each $U_{m}$. This is equivalent to some net in $\{K \in C(X): K \subset X-p\}$ tending to $X$ in the Vietoris topology. We do not need the full definition of the Vietoris topology here.

We call $p \in X$ a cut point to mean $X-p$ is disconnected and a non-cut point otherwise. Clearly each shore point is non-cut. We call $p \in X$ a non-block point to mean $X-p$ has a dense continuum 
component. Every non-block point is a shore point but the converse fails in general [6]. We call $x \in X$ a coastal point to mean $x$ is an element of some proper dense semicontinuum. Clearly $X$ has a non-block point if and only if it has a coastal point.

Theorem 5 of [3] says every point of a metric continuum is coastal. The result generalises to separable continua [1] but not to Hausdorff continua. Under the set-theoretic axiom Near Coherence of Filters the non-metric continuum $\mathbb{H}^{*}$ lacks non-block points and hence lacks coastal points [2].

\section{Equivalent Problems}

Definition 3.1. The continuum $X$ is called partially coastal to mean there are points $x, y \in X$ with $x$ coastal and $y$ non-coastal.

Theorem 1. The following propositions are equivalent.

(1) There exists a continuum without coastal points.

(2) There exists a continuum with exactly one non-coastal point.

(3) There exists a partially coastal continuum.

(4) There exists a continuum without shore points.

(5) There exists a continuum with exactly one shore point

(6) There exists a continuum without non-block points.

(7) There exists a continuum with exactly one non-block point.

Proof. (6) $\Longrightarrow$ (1) because $X$ has a non-block point if and only if it has a coastal point. (2) $\Longrightarrow$ (3) follows from how every continuum has more than one point. (4) $\Longrightarrow$ (6) follows from how every non-block point is a shore point.

$(1) \Longrightarrow(2)$ : Suppose $X$ has no coastal points. Let $[0,1]$ be an arc and the continuum $Y$ be obtained by gluing $1 \in[0,1]$ to any fixed $p \in X$. Identify $X$ and $[0,1]$ with their images in $Y$. The fact each $x \in Y-0$ is coastal is witnessed by the dense semicontinuum $S=(\bigcup\{(1 / n, 1]: n \in$ $\mathbb{N}\}) \cup X=Y-0$.

To see $0 \in Y$ is non-coastal suppose otherwise. That means $0 \in S \subset Y-q$ for some dense proper semicontinuum $S \subset Y$ and point $q \in Y$. It is easy to see $[0,1] \subset S$. Hence $q \notin[0,1]$ and $q \in X-p$. 
Let $Q: Y \rightarrow X$ be the monotone map that collapses $[0,1]$ to the point $p \in X$ and leaves the points of $X$ fixed. Then $Q(S) \subset X$ is a dense semicontinuum with $p \in Q(S) \subset X-q$ contradicting the assumption that $p \in X$ is non-coastal.

$(1) \Longrightarrow(5)$ : Suppose $X$ has no coastal points and define $Y, Q$ and $S$ as before. The dense semicontinuum $S$ witnesses how $0 \in Y$ is a non-block point hence a shore point.

To see 0 is the only shore point first observe each $q \in(0,1]$ is a cut point hence not a shore point. Now suppose some $q \in Y-[0,1]$ is a shore point. Fix the open set $U=(0,1)$. By assumption, for every collection of open sets $U_{1}, \ldots, U_{n} \subset X-1$, some subcontinuum $K \subset Y-q$ meets $U$ and all $U_{m}$. Since $K$ meets $U$ and $U_{1}$ we have $1 \in K$ and hence $p \in Q(K)$.

Now allow $\left\{U_{1}, \ldots, U_{n}\right\}$ to range over all finite collections of open subsets of $X$. The subcontinua $Q(K) \subset X-q$ constitute a dense proper semicontinuum of $X$. Therefore $p \in X$ is coastal contrary to assumption. We conclude $q$ is not a shore point as required. The proof of $(1) \Longrightarrow(7)$ is identical.

(3) $\Longrightarrow$ (4): Suppose some $p \in X$ is non-coastal. Take two disjoint copies $X_{1}$ and $X_{2}$ of $X$. Let $Y$ be the continuum obtained from $X_{1} \sqcup X_{2}$ by identifying the points $a \in X_{1}$ and $b \in X_{2}$ corresponding to $p \in X$. Identify $X_{1}$ and $X_{2}$ with their images in $Y$ and write $p \in Y$ for the shared image of $a$ and $b$. We claim $Y$ has no shore points.

Observe $Y-p$ is homeomorphic to the disjoint union of $X_{1}-a$ and $X_{2}-b$. Hence $p$ is a cut point and not a shore point. Now suppose $q \in Y-p$ is a shore point. Without loss of generality $q \in X_{2}$. Fix some open set $U \subset X_{1}-p$. For every collection of open sets $U_{1}, \ldots, U_{n} \subset X_{2}-p$ some subcontinuum $K \subset Y-q$ meets $U$ and all $U_{m}$. Since $K$ meets $U$ and $U_{1}$ we must have $p \in K$. Moreover $K-p$ is disconnected and each component lies in one of $X_{1}$ or $X_{2}$.

Let $\mathcal{K}$ be the family of components that lie in $X_{2}$. Boundary bumping says $L \cup\{p\}$ is a continuum for each $L \in \mathcal{K}$. It follows $K \cap X_{2}=\bigcup\{\{p\} \cup L: L \in \mathcal{K}\}$ is a subcontinuum of $X_{2}-q$ that meets all $U_{1}, \ldots, U_{n}$. Now allow $\left\{U_{1}, \ldots, U_{n}\right\}$ to range over all finite collections of open subsets of $X_{2}$. Then the union $M$ of all $K \cap X_{2}$ is a dense semicontinuum of $X_{2}-q$. Let $Q: Y \rightarrow X_{2}$ be that map that compresses $X_{1}$ to the point $b \in X_{2}$. Then the semicontinuum $Q(M)$ contradict how $X_{2}$ is not coastal at $b \in X_{2}$. We conclude no $q \in Y$ is a shore point as required.

(5) $\Longrightarrow(1)$ : Suppose $p \in X$ is the unique shore point. We claim $p$ is not coastal, For then $p \in S \subset X-q$ for some $q \in X$ and dense proper semicontinuum $S$. This implies $q$ is a non-block point and hence a shore point contrary to assumption. This implies either (1) or (3) which we have shown are equivalent.

(7) $\Longrightarrow(3)$ : Suppose $p \in X$ is the unique non-block point. Since there is a non-block point there is also a coastal point. But $p \in X$ cannot be coastal as this would imply some $q \in X-p$ is 
a non-block point. hence $X$ is partially coastal.

Under the set-theoretic axiom Near Coherence of Filters (NCF) the Stone-Čech remainder $\mathbb{H}^{*}$ of the half-line is a continuum with no coastal points [2]. From this we get the corollary.

Corollary 3.2. Propositions (1) - (7) from Theorem 1 are consistent.

For each continuum $X$ with a non-coastal point $p$ the author has shown [1] there is a subcontinuum $M \subset X$ with $p \in M^{\circ}$ such that $X / M$ indecomposable and non- coastal at $M$. This raises the question whether $(1)-(7)$ are equivalent if we demand the continuum in question be indecomposable.

Section 5 shows (4) and (5) never hold for indecomposable continua. In particular Theorem 3 says every point of an indecomposable continua is a shore point.

For $X$ indecomposable the remaining propositions seem more difficult and the methods of Theorem 1 no longer apply. For example if $X$ is indecomposable with (1) we cannot simply attach an arc to prove (2) as the quotient space is manifestly decomposable.

In the other direction $X$ having (7) does not imply the same for $X / M$. For example assume NCF and glue any point $p \in \mathbb{H}^{*}$ to the endpoint $1 \in[0,1]$. Let $X$ be the quotient space. Clearly for $X / M$ to be indecomposable $M$ is the union of the arc and some proper subcontinuum $K \subset \mathbb{H}^{*}$ with $p \in K$. Since the map $\mathbb{H}^{*} \rightarrow \mathbb{H}^{*} / K$ is monotone it follows $X / M \cong \mathbb{H}^{*} / K$ lacks non-block points and fails $(7)$.

Before proceeding to Section 5 we take a diversion to prove all shore points are proper shore points. Some of the terminology and results from Section 4 will be needed for Section 5.

\section{Proper Shore and Non-Block Points}

Definition 4.1. Recall $p \in X$ is called a shore point to mean for each finite collection of open sets $U_{1}, U_{2}, \ldots, U_{N} \subset X$ some subcontinuum $K \subset X-p$ meets each $U_{n}$. We call $p$ a proper shore point to mean $K$ can always be chosen as a subset of $\kappa(p)-p$. Otherwise we call $p$ a trivial shore point.

Definition 4.2. Recall $p \in X$ is called a non-block point to mean $X-p$ has a dense continuum component. We call $p$ a proper non-block point to mean $\kappa(p)-p$ has a dense continuum component. Otherwise we call $p$ a trivial non-block point. 
This section shows there exist both proper and trivial non-block points but there is no such thing as a trivial shore point. Thus being a proper non-block point is a meaningful notion but being a proper shore point is not. Hereditarily indecomposable metric continua provide an example of the former.

Lemma 4.3. Suppose $M$ is a hereditarily indecomposable metric continuum. Each $p \in M$ is a trivial non-block point.

Proof. Recall $M$ has $\mathfrak{c}$ many pairwise disjoint composants each of which is a dense semicontinuum [12]. Each composant other than $\kappa(p)$ witnesses how $p$ is a non-block point. It remains to show each continuum component $C$ of $\kappa(p)-p$ is nowhere dense.

Let $q \in C$ be arbitrary. We can write $C=\bigcup \mathcal{C}$ as a union of proper subcontinua with $q \in D$ for each $D \in \mathcal{C}$. Since $q \in \kappa(p)$ there exists a proper subcontinuum $K$ with $\{p, q\} \subset K$. Clearly $K$ meets but is not contained in each $D \in \mathcal{C}$. Hereditary indecomposability implies each $D \subset K$ and therefore $C=\bigcup \mathcal{C} \subset K$. Since $X$ is indecomposable $K$ and thus $C$ is nowhere dense as required.

Observe we only required $M$ to have more than one composant. The problem is open whether there exists a hereditarily indecomposable Hausdorff continuum with exactly one composant. For obstructions to finding such a space see Smith [15, 16, 17, 18].

For $\kappa(p)=X$ clearly the shore point $p$ is proper. Henceforth assume $X$ is irreducible about some $\{p, q\}$. We first treat the case when $X$ is decomposable.

Lemma 4.4. Suppose $X$ is decomposable and irreducible about $\{p, q\}$. Then $p$ and $q$ are proper non-block (shore) points.

Proof. By assumption we can write $X=A \cup B$ as the union of two proper subcontinua. Since $X$ is irreducible we have without loss of generality $p \in A \subset X-q$ and and $q \in B \subset X-p$.

Choose any $x \in A \cap B$. It follows from boundary bumping that $p$ is in the closure of the continuum component $C$ of $x$ in $A-p$. Likewise $q$ is in the closure of the continuum component $D$ of $x$ in $B-q$. Then $x \in C \cup D \subset X-\{p, q\}$ and $C \cup D$ is continuumwise connected.

Observe the subcontinuum $\overline{C \cup D}$ contains $\{p, q\}$. By irreducibility we have $\overline{C \cup D}=X$ hence $C \cup D$ is dense. To see $C \cup D \subset \kappa(p)$ recall $p \in A \subset X-q$ hence $A \cup D \subset \kappa(p)$. Therefore the subset $C \cup D$ of $A \cup D$ witnesses how $p$ is a proper non-block point. By symmetry the same argument applies to $q$.

We now deal with indecomposable $X$. The definition allows finer control over why a given $p \in X$ fails to be a shore point. 
Definition 4.5. Suppose $p \in X$ and $\mathcal{U}=\left\{U_{1}, \ldots, U_{n+1}\right\}$ are open subsets of $X$. We say $p$ disrupts $\mathcal{U}$ to mean no continuum component of $\kappa(p)-p$ meets all $U_{1}, \ldots, U_{n+1}$. We say $p$ trivially disrupts $\mathcal{U}$ to mean there are distinct elements $U^{1}, U^{2}, \ldots, U^{n} \in \mathcal{U}$ and nonempty open sets $V_{i} \subset U^{i}$ such that $p$ disrupts $\left\{V_{1}, \ldots V_{n}\right\}$. Otherwise we say p properly disrupts $\mathcal{U}$.

For $p$ to properly disrupt $\mathcal{U}=\left\{U_{1}, U_{2}, \ldots, U_{n}\right\}$ the definition requires $\mathcal{U}$ be pairwise disjoint. It is also clear the shore point $p \in X$ being trivial is equivalent to disrupting some nondegenerate family. By boundary bumping no $p \in X$ can disrupt a family with only one element. So if $p$ disrupts $\mathcal{U}=\left\{U_{1}, U_{2}\right\}$ then $p$ properly disrupts $\mathcal{U}$.

By induction it follows if $p$ disrupts $\mathcal{U}=\left\{U_{1}, U_{2}, \ldots, U_{n}\right\}$ there exist $m \leq n$ and elements $U^{1}, U^{2}, \ldots, U^{m} \in \mathcal{U}$ and open sets $V_{i} \subset U^{i}$ such that $p$ properly disrupts $\left\{V_{1}, \ldots V_{m}\right\}$. The next lemmas take advantage of that fact.

Lemma 4.6. Suppose $p \in X$ properly disrupts $\left\{U_{1}, \ldots, U_{n}\right\}$ and let $r \leq n$ be fixed. There exists a family $\mathcal{K}(r)$ of subcontinua of $\kappa(p)-p$ that satisfies both properties below.

(1) Each $K \in \mathcal{K}(r)$ meets $U_{m}$ for each $m \neq r$.

(2) $\bigcup \mathcal{K}(r)$ is dense in $U_{m}$ for each $m \neq r$.

Proof. Without loss of generality $r=1$. For each $m \neq 1$ let $V_{m} \subset U_{m}$ be an arbitrary open subset. Since $p$ properly disrupts $\left\{U_{1}, \ldots, U_{n}\right\}$ it cannot disrupt the family $\left\{V_{2}, \ldots, V_{n}\right\}$. That means there is a subcontinuum $K \subset \kappa(p)-p$ that meets each of $V_{2}, \ldots, V_{n}$. Now let $V_{2}, \ldots, V_{n}$ range over the open subsets of $U_{2}, \ldots, U_{n}$. The union of all continua $K$ is dense in each $U_{m}$.

Lemma 4.7. Suppose $X$ is indecomposable. Each shore point $p \in X$ is a proper shore point.

Proof. Suppose to the contrary that $p \in X$ is a trivial shore point. That means $p$ disrupts some family $\mathcal{U}=\left\{U_{1}, \ldots U_{n}\right\}$ of open subsets. By discarding some elements of $\mathcal{U}$ if necessary we can assume $p$ properly disrupts $\mathcal{U}$. Let $\mathcal{K}(1)$ be a family of subcontinua of $\kappa(p)-p$ as described in Lemma4.6.

Each $K \in \mathcal{K}(1)$ has a continuum component $C(K)$ in $\kappa(p)-p$. Since $p$ disrupts $\mathcal{U}$ we know $C(K)$ is disjoint from $U_{1}$. Recall $\overline{C(K)}$ is a subcontinuum. It follows from boundary bumping $p \in \overline{C(K)} \subset X-U_{1}$.

As $K$ ranges over the elements of $\mathcal{K}(1)$ the set $S=\bigcup\{\overline{C(K)}: K \in \mathcal{K}(1)\}$ constitutes a semicontinuum with $p \in S \subset X-U_{1}$. Therefore $\bar{S} \subset X-U_{1}$ is a proper subcontinuum. But Property (2) of Lemma 4.6 says $U_{2} \subset \bar{S}$. Thus $\bar{S} \subset X$ is a proper subcontinuum with nonvoid interior. Since $X$ is indecomposable this cannot occur. We conclude $p$ is a proper shore point. 
Theorem 2 follows from Lemmas 4.4 and 4.7 .

Theorem 2. Shore points are the same as proper shore points.

\section{Continua Without Shore Points}

The proof of Theorem 1 says we can build a continuum $X$ without shore points by assuming NCF and gluing two copies of $\mathbb{H}^{*}$ together at a single point. This section is about whether arbitrary continua $X$ without shore points come about this way - by joining together continua without non-block points. We first show $X$ is decomposable.

Lemma 5.1. Suppose $p \in X$ is not a shore point. There is a decomposition $X=X_{1} \oplus \ldots \oplus X_{n}$ with $p \in X_{1} \cap \ldots \cap X_{n}$.

Proof. Since $p$ is not a shore point it disrupts some family $\mathcal{U}=\left\{U_{1}, \ldots U_{n}\right\}$ of open subsets. Like before, we can assume $p$ properly disrupts $\mathcal{U}$. For each $r \leq n$ let $\mathcal{K}(r)$ be a family of subcontinua of $\kappa(p)-p$ as described in Lemma 4.6,

The proof of Lemma4.7 shows each $K(r)=\overline{\bigcup \mathcal{K}(r)}$ is a subcontinuum with $p \in K(r) \subset X-U_{r}$ and $U_{m} \subset K(r)$ for each $m \neq r$. Let $\mathcal{L}(r)$ be the family of all subcontinua with both properties.

Without loss of generality we can assume $K(r)=\overline{\bigcup \mathcal{L}(r)}$ is the largest subcontinuum with both properties.

Define the subcontinuum $Y=K(1) \cup K(2) \cup \ldots \cup K(n)$ and write $C(x)$ for the continuum component of each $x \in X-Y$. We claim each $C(x)$ meets no $U_{r}$. For suppose otherwise. Then without loss of generality some $C(x)$ meets $U_{1}$. Since $p$ disrupts $\left\{U_{1}, \ldots U_{n}\right\}$ we know $C(x)$ fails to meet at least one $U_{m}$. Without loss of generality $m=2$. Then $\overline{C(x)}$ is a subcontinuum with $x \in \overline{C(x)} \subset X-U_{2}$.

Now consider the subcontinuum $K(2) \cup \overline{C(x)}$. We have $p \in K(2) \cup \overline{C(x)} \subset X-U_{2}$ and $U_{m} \subset K(2) \cup \overline{C(x)}$ for each $m \neq 2$. Since we chose $K(2)$ to be maximal with both properties we must have $K(2) \cup \overline{C(x)}=K(2)$. Hence $\overline{C(x)} \subset K(2)$ and $x \in K(2) \subset Y$ contrary to the assumption that $x \in X-Y$. We conclude $C(x)$ meets no $U_{r}$.

It follows for each $x \in X-Y$ that $\overline{C(x)}$ is a subcontinuum with $\{p, x\} \subset \overline{C(x)} \subset X-\left(U_{1} \cup \ldots \cup\right.$ $\left.U_{n}\right)$. Define the subcontinuum $C=\overline{\bigcup\{C(x): x \in X-Y\}}$. Then $\{p, x\} \subset C \subset X-\left(U_{1} \cup \ldots \cup U_{n}\right)$.

It follows $X=C \cup K(1) \cup K(2) \cup \ldots \cup K(n)$ and $p$ is an element of each element of the covering. By discarding any covering element contained in the union of the others we get the required decomposition. 
The next theorem follows from Theorem 2 and Lemma 5.1 ,

Theorem 3. Every point of an indecomposable continuum is a proper shore point.

The proof of Theorem 3 for the special case of $\mathbb{H}^{*}$ uses standard techniques from the study of the Stone-Čech remainder. An earlier paper of ours gave a lengthy argument for $\mathbb{H}^{*}$, that considered separately two types of composants of $\mathbb{H}^{*}$ and did not use Theorem 2 . That paper was never published because the anonymous referee was able to give the much simpler proof we include here as Lemma 5.2. The second half of the proof is based on [5] Theorem 4.1.

We briefly recall the terminology from [2]: The Stone-Čech remainder $\mathbb{H}^{*}$ is the set of nonprincipal ultrafilters of closed sets on the half open interval $\mathbb{H}=[0, \infty)$. The topology on $\mathbb{H}^{*}$ is generated by the sets

$$
U^{*}=\left\{\mathcal{D} \in \mathbb{H}^{*}: D \subset U \text { for some } D \in \mathcal{D}\right\}
$$

as $U$ ranges over all open subsets of $\mathbb{H}$. Note for $U$ bounded we have $U^{*}=\varnothing$. It is known that $\mathbb{H}^{*}$ is an indecomposable Hausdorff continuum.

Suppose we have a nonprincipal ultrafilter $\mathcal{D}$ on $\omega$ and sequence of intervals $I_{n}=\left[a_{n}, b_{n}\right]$ with each $b_{n}<a_{n+1}$. For each subset $D \subset \omega$ we write $I_{D}=\bigcup\left\{I_{n}: n \in D\right\}$ for the subset of $\mathbb{H}$. We write $\overline{I_{D}}$ for the closure in $\beta \mathbb{H}$. That means the collection of ultrafilters $\mathcal{D}$ on $\mathbb{H}$ with $I_{D} \in \mathcal{D}$. We write $\mathbb{I}_{\mathcal{D}}$ for the subset $\bigcap\left\{\mathbb{H}^{*} \cap \overline{I_{D}}: D \in \mathcal{D}\right\}$ of $\mathbb{H}^{*}$. Sets of the form $\mathbb{I}_{\mathcal{D}}$ are known to be proper subcontinua of $\mathbb{H}^{*}$ and are called standard subcontinua. For background on $\mathbb{H}^{*}$ see [9]. For background on Stone-Čech compactifications in general see [7] and [19].

Lemma 5.2. Let $p \in \mathbb{H}^{*}$ be arbitrary and $\mathcal{U}=\left\{U_{1}, \ldots, U_{n}\right\}$ a family of open sets. For each standard subcontinuum $\mathbb{I}_{\mathcal{D}}$ with $p \in \mathbb{I}_{\mathcal{D}}$ some other standard subcontinuum $\mathbb{J}_{\mathcal{D}} \subset \kappa(p)$ has $p \notin \mathbb{J}_{\mathcal{D}}$ but $\mathbb{J}_{\mathcal{D}}$ meets each $U_{m}$. In particular each point of $\mathbb{H}^{*}$ is a shore point.

Proof. We only consider $n=2$ and $\mathcal{U}=\left\{U_{1}, U_{2}\right\}$ as the general case is similar. First choose disjoint open $U, V \subset \mathbb{H}$ with the basic open sets $U^{*} \subset U_{1}$ and $V^{*} \subset U_{2}$. Then observe $U=$ $A_{1} \cup A_{2} \cup \ldots$ is a disjoint union of open intervals and likewise for $V=B_{1} \cup B_{2} \cup \ldots$.

Choose an increasing sequence $c_{1}<c_{2}<\ldots$ in $\mathbb{H}$ so each $\left[c_{n}, c_{n+1}\right]$ contains at least one $A_{i}$ and $B_{j}$. It follows $c_{n} \rightarrow \infty$. Define sequences $R_{n}=\left[c_{4 n}, c_{4 n+1}\right]$ and $L_{n}=\left[c_{4 n+2}, c_{4 n+3}\right]$. By construction $\mathbb{R}_{\mathcal{D}}$ and $\mathbb{L}_{\mathcal{D}}$ are disjoint and each meets $U^{*}$ and $V^{*}$. Therefore $p$ is an element of at most one of $\mathbb{R}_{\mathcal{D}}$ and $\mathbb{L}_{\mathcal{D}}$. Without loss of generality $p \notin \mathbb{R}_{\mathcal{D}}$.

Taking $\mathbb{J}_{\mathcal{D}}=\mathbb{R}_{\mathcal{D}}$ it remains to show $\mathbb{J}_{\mathcal{D}} \subset \kappa(p)$. In case $\mathbb{J}_{\mathcal{D}}$ meets $\mathbb{I}_{\mathcal{D}}$ the fact that $\mathbb{H}^{*}$ is indecomposable says $\mathbb{J}_{\mathcal{D}} \cup \mathbb{I}_{\mathcal{D}} \neq \mathbb{H}^{*}$ hence $\mathbb{J}_{\mathcal{D}} \subset \kappa(p)$ as required. Otherwise $\mathbb{I}_{\mathcal{D}}$ and $\mathbb{J}_{\mathcal{D}}$ are 
disjoint. Recall $\mathbb{I}_{\mathcal{D}}=\bigcap\left\{\mathbb{H}^{*} \cap \overline{I_{D}}: D \in \mathcal{D}\right\}$ and $\mathbb{J}_{\mathcal{D}}=\bigcap\left\{\mathbb{H}^{*} \cap \overline{J_{D}}: D \in \mathcal{D}\right\}$ are intersections of closed subsets of the compact $\mathbb{H}^{*}$. Since they are disjoint the Cantor property says $\mathbb{H}^{*} \cap \overline{I_{A}}$ and $\mathbb{H}^{*} \cap \overline{J_{B}}$ are disjoint for some $A, B \in \mathcal{D}$.

It follows from the definition of $\overline{I_{A}}$ and $\overline{I_{B}}$ that $I_{A} \cap J_{B} \subset \mathbb{H}$ is compact. Since $A \cap B \in \mathcal{D}$ and $I_{A \cap B} \cap J_{A \cap B} \subset I_{A} \cap J_{B}$ we also see $I_{A \cap B} \cap J_{A \cap B}$ is compact. That means the set

$$
C=\left\{n \in A \cap B: I_{n} \text { meets } J_{m} \text { for some } m \in A \cap B\right\}
$$

is finite. Since $\mathcal{D}$ is nonprincipal $C \notin \mathcal{D}$ and so $D=(A \cap B-C) \in \mathcal{D}$.

According to the definiton of $\mathbb{I}_{\mathcal{D}}$ as in intersection, the subcontinuum $\mathbb{I}_{\mathcal{D}}$ does not change if we redefine the intervals $I_{n}$ for all $n \in D^{c}$. Likewise for $\mathbb{J}_{\mathcal{D}}$. Hence we can redefine $I_{n}, J_{n}$ for $n \in D^{c}$ without changing $\mathbb{I}_{\mathcal{D}}$ and $\mathbb{J}_{\mathcal{D}}$ and hence assume $\mathbb{I}_{\omega} \cap \mathbb{J}_{\omega}$ is empty. Then we can combine the two sequences into some $\left\{K_{n}: n \in \omega\right\}$ where each interval $K_{n}$ is some $I_{m}$ or $J_{m}$ and each $K_{n+1}$ is to the right of $K_{n}$.

Define the maps $\alpha: \omega \rightarrow \omega$ by letting each $\alpha(n)$ be the unique $i \in \omega$ with $I_{n}=K_{i}$. In other words $I_{n}=K_{\alpha(n)}$. Likewise define $\beta: \omega \rightarrow \omega$ by $J_{n}=K_{\beta(n)}$. It follows from the definitions that $\mathbb{I}_{\mathcal{D}}=\mathbb{K}_{\alpha(\mathcal{D})}$ and $\mathbb{J}_{\mathcal{D}}=\mathbb{K}_{\beta(\mathcal{D})}$ where we define the ultrafilter $\alpha(\mathcal{D})=\left\{E \subset \omega: \alpha^{-1}(E) \in \mathcal{D}\right\}$ and likewise for $\beta(\mathcal{D})$.

Define a finite-to-one function $g$ separately over the disjoint sets $A=\{\alpha(n): n \in \omega\}$ and $B=\{\beta(n): n \in \omega\}$ by $g(\alpha(n))=\alpha(n)$ and $g(\beta(n))=\alpha(n)$. The definition $g(\beta(n))=\alpha(n)$ makes sense because $\beta$ is injective. From here it quickly follows $g(\alpha(\mathcal{D}))=\alpha(\mathcal{D})=g(\beta(\mathcal{D}))$. From [4] Lemma 10 and the paragraph before that lemma, if there exists a finite-to-one function $g: \omega \rightarrow \omega$ with $g(\alpha(\mathcal{D}))=g(\beta(\mathcal{D}))$ then there also exists a finite-to-one monotone function $f: \omega \rightarrow \omega$ with $f(\alpha(\mathcal{D}))=f(\beta(\mathcal{D}))$.

Since $f$ is finite-to-one each $f^{-1}(n)$ is finite. Thus the convex hull $L_{n}$ of $\left\{K_{m}: m \in f^{-1}(n)\right\}$ is a closed interval. Since $f$ is monotone each $L_{n+1}$ is to the right of $L_{n}$. So we can define a standard subcontinuum $\mathbb{L}_{f(\alpha(\mathcal{D}))}$. We claim $\mathbb{K}_{\alpha(\mathcal{D})}, \mathbb{K}_{\beta(\mathcal{D})} \subset \mathbb{L}_{f(\alpha(\mathcal{D}))}$. Since $\mathbb{J}_{\mathcal{D}}=\mathbb{K}_{\beta(\mathcal{D})}$ this shows $\mathbb{J}_{\mathcal{D}} \subset \kappa(p)$.

To that end write $\alpha(\mathcal{D})=\mathcal{U}$. First observe each $K_{n} \subset L_{f(n)}$ since $L_{f(n)}$ is the hull of $\left\{K_{m}\right.$ : $\left.m \in f^{-1}(f(n))\right\}$ and we have $m \in f^{-1}(f(n))$ for $m=n$. It follows $K_{U} \subset L_{f(U)}$ for each $U \in \mathcal{U}$. Hence we can write

$$
\mathbb{K}_{\mathcal{U}}=\bigcap_{U \in \mathcal{U}} \mathbb{H}^{*} \cap \overline{K_{U}} \subset \bigcap_{U \in \mathcal{U}} \mathbb{H}^{*} \cap \overline{L_{f(U)}}
$$

It follows from the definition of $f(\mathcal{U})$ and $\mathcal{U}$ being an ultrafilter that $f(U) \in f(\mathcal{U})$ for each 
$U \in \mathcal{U}$. In the other direction, for each $E \in f(\mathcal{U})$ we have $f^{-1}(E) \in \mathcal{U}$ and so $E$ contains the set $f(U)$ for $U=f^{-1}(E)$. It follows $\{f(U): U \in \mathcal{U}\}$ is a cofinal subset of $f(\mathcal{U})$. Hence the intersection on the right-hand-side equals

$$
\bigcap_{W \in f(\mathcal{U})} \mathbb{H}^{*} \cap \overline{L_{W}}=\mathbb{L}_{f(\mathcal{U})} .
$$

We conclude $\mathbb{K}_{\alpha(\mathcal{D})} \subset \mathbb{L}_{f(\alpha(\mathcal{D}))}$. By symmetry the same holds for $\mathbb{K}_{\beta(\mathcal{D})}$. This completes the proof.

We would like to show our example of spot-welding two copies of $\mathbb{H}^{*}$ is generic in the following sense.

Conjecture 4. Suppose $X$ has no shore points and $p \in X$. There is a decomposition $X=X_{1} \oplus$ $\ldots \oplus X_{N}$ with $p \in X_{1} \cap \ldots \cap X_{N}$ and $p$ non-coastal when treated as a point of each $X_{n}$.

Conjecture 4 asks for a particularly nice decomposition of $X$. One variant of the conjecture is that every decomposition with $p \in X_{1} \cap \ldots \cap X_{N}$ is nice. The stronger conjecture however is false.

For a counterexample assume NCF and take two copies $H_{1}$ and $H_{2}$ of $\mathbb{H}^{*}$ and identify points $x_{1} \in H_{1}$ and $x_{2} \in H_{2}$ respectively with the endpoints 0 and 1 of the arc. Denote by $X$ the quotient space.

Observe for $X_{1}=H_{1} \cup[0,1]$ and $X_{2}=[0,1] \cup H_{2}$ we have the decomposition $X=X_{1} \oplus X_{2}$ but the semicontinua $H_{1} \cup[0,1)$ and $(0,1] \cup H_{2}$ witness how $p=1 / 2$ is a coastal point of each element. Thus the stronger conjecture fails.

To see the weaker conjecture holds consider the second choice of decomposition $X_{1}=H_{1} \cup$ $[0,1 / 2]$ and $X_{2}=[1 / 2,1] \cup H_{2}$. By the same reasoning as in Theorem 1 we see $p=1 / 2$ is a coastal point of neither element.

The second decomposition above is minimal in the following sense.

Definition 5.3. Suppose $X=X_{1} \oplus \ldots \oplus X_{N}$ and $X=Y_{1} \oplus \ldots \oplus Y_{N}$ are decompositions. We write $Y_{1} \oplus \ldots \oplus Y_{N} \leq X_{1} \oplus \ldots \oplus X_{N}$ to mean there is a permutation $\sigma$ of $\{1,2, \ldots, N\}$ with each $Y_{n} \subset X_{\sigma(n)}$. We call a decomposition minimal to mean it is minimal with respect to this partial order.

For example, each minimal decomposition $X_{1} \oplus X_{2}$ of the arc has $X_{1} \cap X_{2}$ a singleton. Each minimal decomposition $X_{1} \oplus X_{2}$ of the circle has $X_{1} \cap X_{2}$ a doubleton. For $X$ formed by spotwelding finitely many indecomposable continua the natural decomposition is minimal. It may prove useful that minimal decompositions always exist. 
Lemma 5.4. Each decomposition is $\leq$-above some minimal decomposition.

Proof. The proof uses Zorn's lemma. Suppose $\left\{X^{i}(1) \oplus \ldots \oplus X^{i}(N): i \in \mathcal{I}\right\}$ is a chain of decompositions. Without loss of generality $\mathcal{I}$ has top element $1 \in \mathcal{I}$ and no bottom element. Since $X^{1}(1) \oplus \ldots \oplus X^{1}(N)$ is a decomposition there are points $x_{n} \in X^{1}(n)-\bigcup\left\{X^{1}(m): m \neq n\right\}$.

Let $X^{i}(1) \oplus \ldots \oplus X^{i}(N)$ be arbitrary. Since $i \leq 1$ some permutation $\sigma_{i}$ has each $X^{i}\left(\sigma_{i}(n)\right) \subset$ $X^{1}(n)$. Thus $X^{i}\left(\sigma_{i}(n)\right)$ includes at most the element $x_{n}$ of $\left\{x_{1}, x_{2}, \ldots, x_{N}\right\}$. But since $X^{i}(1) \cup$ $\ldots \cup X^{i}(N)=X$ each $x_{n}$ is an element of some $X^{i}(m)$. We conclude each $x_{n} \in X^{i}\left(\sigma_{i}(n)\right)-$ $\bigcup\left\{X^{i}\left(\sigma_{i}(m)\right): m \neq n\right\}$.

For $j \leq i$ we know $X^{j}\left(\sigma_{j}(n)\right)$ is contained in some $X^{i}\left(\sigma_{i}(m)\right)$. The point $x_{n}$ witnesses how $m=n$. We conclude each $X^{j}\left(\sigma_{j}(m)\right) \subset X^{i}\left(\sigma_{i}(m)\right)$. Then [14] Proposition 1.7 says the intersection $X_{n}=\bigcap\left\{X^{i}\left(\sigma_{i}(n)\right): i \in I\right\}$ is a subcontinuum. We claim $X_{1}, \ldots, X_{N}$ form a decomposition which is clearly a lower bound for the chain. First observe each $x_{n}$ witnesses how $X_{n}$ is not contained in $\bigcup\{X(m): m \neq n\}$.

To show $X_{1} \cup \ldots \cup X_{N}=X$ let $x \in X$ be arbitrary. For each $i \in \mathcal{I}$ there is $n_{i} \leq N$ with $x \in X^{i}\left(\sigma_{i}\left(n_{i}\right)\right)$. Write $A(n)=\left\{i \in \mathcal{I}: x \in X^{i}\left(\sigma_{i}(n)\right)\right\}$ for $n=1,2, \ldots, N$. Since $\mathcal{I}$ has no bottom element one of $A(n)$ is cofinal. Thus $x \in \bigcap\left\{X^{i}\left(\sigma_{i}(n)\right): i \in A(n)\right\}$ which equals $\bigcap\left\{X^{i}\left(\sigma_{i}\left(n_{i}\right)\right): i \in \mathcal{I}\right\}$ by cofinality and thus $x \in X_{n}$. Since $x$ is arbitrary we see $X_{n}$ cover $X$.

We conclude the chain $\left\{X^{i}(1) \oplus \ldots \oplus X^{i}(N): i \in \mathcal{I}\right\}$ has a lower bound $X_{1} \oplus \ldots \oplus X_{N}$. Zorn's lemma then implies each decomposition is above a minimal decomposition.

Conjecture 5. Suppose $X$ has no shore points and $X=X_{1} \oplus \ldots \oplus X_{N}$ is a minimal decomposition with $p \in X_{1} \cap \ldots \cap X_{N}$. Then $p$ is non-coastal when treated as a point of each $X_{n}$.

Thus far we have only the partial results Lemmas 5.5, 5.8 and 5.9. Henceforth assume $X$ has no shore points. Fix $p \in X$ and let $X=X_{1} \oplus X_{2}$ be a decomposition with $p \in X_{1} \cap X_{2}$. The case for $n>2$ is similar.

Lemma 5.5. Each dense semicontinuum of $X_{1}\left(\right.$ resp. $\left.X_{2}\right)$ at $p$ contains $X_{1}-X_{2}\left(\right.$ resp. $\left.X_{2}-X_{1}\right)$.

Proof. Suppose for example $p \in S \subset X_{1}-q$ for some dense semicontinuum $S \subset X_{1}$ and $q \in$ $X_{1}-X_{2}$. Then $X_{2} \cup S \subset X-q$ is a dense semicontinuum of $X$. This implies $q \in X$ is a non-block point hence a shore point contrary to assumption.

Corollary 5.6. Suppose $X_{1} \cap X_{2}=\{p\}$. Then $p$ is non-coastal as an element of $X_{1}$ and $X_{2}$. 
The following notation is part of Lemma 5.8

Notation 5.7. Suppose $X=X_{1} \oplus X_{2}$. Write $\mathcal{S}\left(X_{1}\right)$ (resp. $\mathcal{S}\left(X_{2}\right)$ ) for the collections of proper dense semicontinua of $X_{1}$ (resp. $X_{2}$ ) that meet both $X_{1} \cap X_{2}$ and $X_{1}-X_{2}$ (resp. $X_{2}-X_{1}$ ). Define two subsets of $X_{1} \cap X_{2}$.

$$
\begin{aligned}
& C_{1}=\left\{x \in X_{1} \cap X_{2}: x \in S \text { for each } S \in \mathcal{S}\left(X_{1}\right)\right\} . \\
& C_{2}=\left\{x \in X_{1} \cap X_{2}: x \in S \text { for each } S \in \mathcal{S}\left(X_{2}\right)\right\} .
\end{aligned}
$$

Lemma 5.8. Suppose $p$ is coastal as an element of both $X_{1}$ and $X_{2}$. Then one of $C_{1} \cap C_{2}=\varnothing$ or $C_{1} \cup C_{2}=X_{1} \cap X_{2}$ holds.

Proof. Since $p \in X_{1}$ is coastal $\mathcal{S}\left(X_{1}\right)$ is nonempty and likewise for $\mathcal{S}\left(X_{2}\right)$. Suppose $C_{1} \cap C_{2} \neq \varnothing$ and $C_{1} \cup C_{2} \neq X_{1} \cap X_{2}$. That means there are $x \in C_{1} \cap C_{2}$ and $y \in X_{1} \cap X_{2}-C_{1} \cup C_{2}$. Select $S_{1} \in \mathcal{S}\left(X_{1}\right)$ and $S_{2} \in \mathcal{S}\left(X_{2}\right)$ with $y \notin S_{1}$ and $y \notin S_{2}$.

By definition we have $x \in S_{1}$ and $x \in S_{2}$. Thus $S_{1} \cup S_{2} \subset X$ is a dense semicontinuum that excludes the point $y \in X$. This contradicts how $X$ has no shore points. We conclude $C_{1} \cap C_{2} \neq \varnothing$ and so $C_{1} \cup C_{2}=X_{1} \cap X_{2}$.

In the first case of Lemma 5.8 we can say more.

Lemma 5.9. Suppose $p$ is coastal as an element of both $X_{1}$ and $X_{2}$ and $C_{1} \cap C_{2}=\varnothing$. Then each subcontinuum of $X$ that meets $C_{1}$ and $C_{2}$ also contains $X_{1} \cap X_{2}-C_{1} \cup C_{2}$.

Proof. Suppose the subcontinuum $K \subset X$ meets $C_{1}$ and $C_{2}$. Let $x \in X_{1} \cap X_{2}-C_{1} \cup C_{2}$ be arbitrary. Select $S_{1} \in \mathcal{S}\left(X_{1}\right)$ and $S_{2} \in \mathcal{S}\left(X_{2}\right)$ with $x \notin S_{1}$ and $x \notin S_{2}$. It follows $S_{1} \cup K \cup S_{2} \subset X$ is a dense semicontinuum. Since $X$ has no shore points $S_{1} \cup K \cup S_{2}=X$ and so $x \in K$. Since $x \in X_{1} \cap X_{2}-C_{1} \cup C_{2}$ is arbitrary we conclude $X_{1} \cap X_{2}-C_{1} \cup C_{2} \subset K$.

\section{Acknowledgements}

This research was supported by the Irish Research Council Postgraduate Scholarship Scheme grant number GOIPG/2015/2744. The author would like to thank Professor Paul Bankston and Doctor Aisling McCluskey for their help in preparing the manuscript, and the anonymous referee for their attention and suggestions. 


\section{References}

[1] Daron Anderson. Shore and non-block points in Hausdorff continua. Topology and its Applications, 193:152 - 161, 2015.

[2] Daron Anderson. A Continuum Without Non-Block Points. Topology and its Applications, 218:42 - 52, 2017.

[3] R. H. Bing. Some characterizations of arcs and simple closed curves. American Journal of Mathematics, 70(3):pp. 497-506, 1948.

[4] Andreas Blass. Near coherence of filters. I: Cofinal equivalence of models of arithmetic. Notre Dame J. Formal Logic, 27(4):579-591, 101986.

[5] Andreas Blass. Near coherence of filters. II: Applications to operator ideals, the Stone-Čech remainder of a half-line, order ideals of sequences, and slenderness of groups. Transactions of the American Mathematical Society, 300(2):pp. 557-581, 1987.

[6] Jozef Bobok, Pavel Pyrih, and Benjamin Vejnar. Non-cut, shore and non-block points in continua. Glasnik Matematicki, 51(1):237 - 253, 2016.

[7] W.W. Comfort and S. Negrepontis. The Theory of Ultrafilters. Springer-Verlag, 1974.

[8] Ryszard Engelking. General Topology. Heldermann Verlag Berlin, 1989.

[9] K.P Hart. The Čech-Stone compactification of the real line. In M. Hušek and J. van Mill, editors, Recent Progress in General Topology. North Holland, 1992.

[10] Kazimierz Kuratowski. Topology Volume II. Academic Press, 1968.

[11] Rocío Leonel. Shore points of a continuum. Topology and its Applications, 161(0):433 $441,2014$.

[12] Stefan Mazurkiewicz. Sur les continus indécomposables. Fundamenta Mathematicae, 10(1):305-310, 1927.

[13] Robert L. Moore. Concerning the cut-points of continuous curves and of other closed and connected point-sets. Proceedings of the National Academy of Sciences of the United States of America, 161(4):101 - 106, 1923.

[14] Sam B. Nadler Jr. Continuum Theory: An Introduction. CRC Press, 1992. 
[15] Michel Smith. No arbitrary product of $\beta[0, \infty)-[0, \infty)$ contains a nondegenerate hereditarily indecomposable continuum. Topology and its Applications, 28(1):23 - 28, 1988.

[16] Michel Smith. Hereditarily indecomposable subcontinua of the inverse limit of lexicographic arcs are metric. Topology and its Applications, 154(15):2813 - 2816, 2007.

[17] Michel Smith. Hereditarily indecomposable subcontinua of inverse limits of Souslin arcs are metric. Topology and its Applications, 155(6):540 - 543, 2008.

[18] Michel Smith. Hereditarily indecomposable subcontinua of the product of two Souslin arcs are metric. Topology and its Applications, 156(3):522 - 524, 2009.

[19] Russell C. Walker. The Stone-Čech Compactification. Springer-Verlag New York, 1974. 